\title{
Potencial del uso de la radiografía panorámica como ayuda diagnóstica en derechohabientes de una institución de seguridad social.
}

\author{
Potential for the use of panoramic radiography as a diagnostic \\ aid in beneficiaries of a social security institution. \\ José Alan Villalobos-Mendoza,* Gustavo Adolfo Guerra,* Luz Helena Sanin, \\ Raúl Eduardo Baeza-Rubio, ${ }^{\S}$ Nydia Elisa Hinojos-Villalobos*
}

\section{RESUMEN}

Introducción: A pesar de que actualmente la radiografía panorámica es un instrumento auxiliar de diagnóstico de uso común, desafortunadamente sus beneficios no se aprovechan en su totalidad y su empleo se limita al uso en determinadas áreas de la odontología y en ocasiones se omiten hallazgos que pueden tener un significado clínico importante. Material y métodos: Estudio transversal y descriptivo de una muestra aleatoria de 500 estudios de imagen (radiografías panorámicas) durante el periodo de enero a mayo de 2018 analizadas por expertos estandarizados y empleando estadística descriptiva básica mediante el paquete estadístico Excel. Resultados: Se incluyeron 500 estudios, $67 \%$ correspondió a mujeres en un rango de cinco a 91 años de edad con una mediana de 43 años, $48 \%$ presentó alteraciones siendo las más frecuentas pérdida del proceso alveolar $45 \%$, alteraciones en articulación temporomandibular 34\%, y calcificación del ligamento estilohioideo $31 \%$. Conclusión: Se reportaron hallazgos clínico-radiográficos significativos, resaltando la necesidad de un análisis cuidadoso de los métodos auxiliares de diagnóstico que permitan visualizar de manera contextual el tratamiento odontológico de los pacientes y/o reportar al especialista correspondiente otro tipo de hallazgos.

Palabras clave: Auxiliar, diagnóstico, ortopantomografía, México.

\section{ABSTRACT}

Introduction: Although panoramic radiography is currently a commonly used diagnostic auxiliary instrument, unfortunately its benefits are not fully exploited and its use is limited to use in certain areas of Dentistry and eventually findings that may have important clinical significance are omitted. Material and methods: Crosssectional and descriptive study of a random sample of 500 imaging studies (panoramic radiographs) during the period from January to May 2018, analyzed by standardized experts and using basic descriptive statistics using the Excel statistical package. Results: 500 studies were included corresponding to $67 \%$ women and an age range from five to 91 years of age with a median of 43 years, $48 \%$ presented alterations, the most frequent being loss of the alveolar process $45 \%$, alterations in the temporomandibular joint $34 \%$, and calcification of the hyoid ligament $31 \%$. Conclusion: In this study significant clinical-radiographic findings are reported, highlighting the need for a careful analysis of auxiliary diagnostic methods that allow visualize the dental treatment of the patients and / or report other findings to the corresponding specialist.

Keywords: Auxiliary, diagnosis, orthopantomography, Mexico.

\footnotetext{
* Facultad de Odontología, Universidad Autónoma de Chihuahua, Chihuahua, México.

₹ Facultad de Medicina, Universidad Autónoma de Chihuahua, Chihuahua, México.

$\S$ Pensiones Civiles del Estado de Chihuahua, Chihuahua, México.

Recibido: 24 de junio de 2020. Aceptado: 16 de agosto de 2021.
}

Citar como: Villalobos-Mendoza JA, Guerra GA, Sanin LH, Baeza-Rubio RE, Hinojos-Villalobos NE. Potencial del uso de la radiografía panorámica como ayuda diagnóstica en derechohabientes de una institución de seguridad social. Rev ADM. 2021; 78 (6): 346-349. https://dx.doi.org/10.35366/102976 


\section{INTRODUCCIÓN}

L a radiografía panorámica $(\mathrm{RP})$ se usa regularmente como ayuda en el diagnóstico y plan de tratamiento de los pacientes en el área odontológica. Es un procedimiento extraoral simplificado que permite visualizar toda la región maxilomandibular en una única película, ya que facilita el examen de toda la dentición, hueso alveolar, articulación temporomandibular y estructuras adyacentes. ${ }^{1}$ Además de obtenerse con rapidez, la toma de la técnica radiográfica es más cómoda y de menor radiación para el paciente y para el profesional de la salud. ${ }^{2}$ La RP nos permite mejorar el diagnóstico, pronóstico y tratamiento de entidades patológicas tales como lesiones patológicas, dientes supernumerarios o impactados, alteraciones en los senos maxilares, fosas nasales, lesiones osteolíticas y osteogénicas en el maxilar y mandíbula. ${ }^{1,3}$

Existen distintas alteraciones en la cavidad oral y estructuras del complejo de cabeza y cuello, cuyo hallazgo y/o diagnóstico sólo se puede realizar por medio de un análisis imagenológico, ya sean radiografías panorámicas, periapicales $\mathrm{u}$ otros exámenes auxiliares.

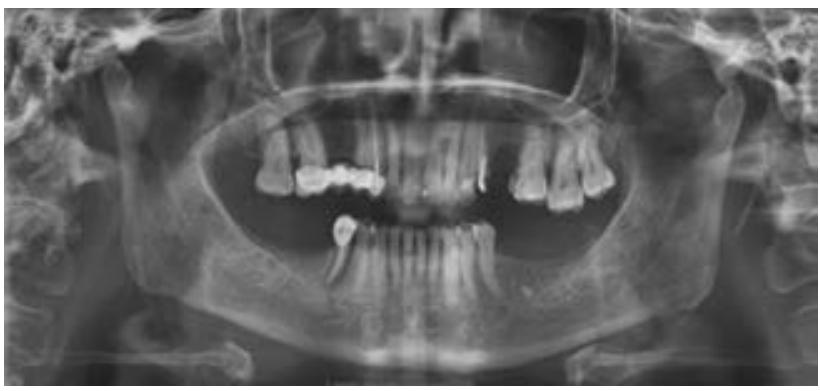

Figura 1: Manifiesta elongación del proceso estilohioideo, pérdida del proceso alveolar. En zona articular presenta asimetría de en cóndilo articular izquierdo.

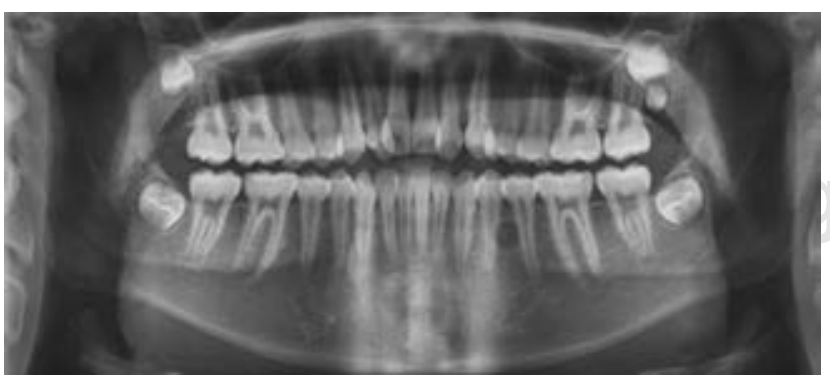

Figura 2: Zona radiolúcida a nivel de pieza 35 con bordes escleróticos, a nivel de pieza 27 se observa órgano dental supernumerario.
Tabla 1: Número de casos y porcentaje de hallazgos radiográficos con mayor presencia en el estudio.

\begin{tabular}{lc} 
Hallazgos radiográficos & $\mathrm{n}(\%)$ \\
\hline $\begin{array}{l}\text { Pérdida de ósea proceso alveolar } \\
\text { Alteración de zona articular (ATM) }\end{array}$ & $226(45)$ \\
$\begin{array}{l}\text { Calcificación del ligamento estilohioideo } \\
\text { (CLE-síndrome de Eagle) }\end{array}$ & $171(34)$ \\
$\begin{array}{l}\text { Zonas radiopacas a nivel de carótida } \\
\text { (calcificaciones de carótida) }\end{array}$ & $181(30)$ \\
Zonas radiolúcidas & $11(2)$ \\
\hline
\end{tabular}

El profesional de la salud oral debe ser capaz de detectar anomalías y patologías tempranamente para llegar a un diagnóstico oportuno mediante esta técnica, estableciendo parámetros para la evaluación y valoración radiográfica con el fin de logar un manejo integral de los pacientes. . $^{4}$

Basados en la descripción de los síntomas del paciente, así como de la examinación, podemos determinar qué tipo de herramientas diagnósticas son las más apropiadas para confirmar un diagnóstico y desarrollar un plan de tratamiento adecuado. ${ }^{6}$ El presente estudio se realizó con la finalidad de analizar las posibilidades diagnósticas de esta técnica.

\section{MATERIAL Y MÉTODOS}

Se llevó a cabo un estudio de análisis tipo retrospectivo descriptivo en una institución de seguridad social utilizando una muestra aleatoria de 500 pacientes durante el periodo del 05 de enero al 31 de mayo de 2018. Se incluyeron los pacientes derechohabientes de la institución que tuvieran información clínica completa así como $\mathrm{RP}$ reciente. De las historias clínicas se obtuvieron datos demográficos (género, edad, enfermedades sistémicas). Las RP se efectuaron mediante el equipo Vatech PAXISC (Corea). El análisis de las RP fue realizado por tres odontólogos calibrados previamente para tal efecto: de las radiografías impresas se tomaron imágenes en formato jpeg, las cuales se dividieron en cuatro cuadrantes: superior derecho, superior izquierdo, inferior izquierdo e inferior derecho, registrando los hallazgos por cuadrante en una base de datos de Excel.

\section{RESULTADOS}

De los 500 pacientes incluidos en este estudio, 336 $(67 \%)$ pacientes correspondieron al género femenino 
y $164(33 \%)$ al masculino, con una media de edad de 43 años (mínimo de cinco años y máximo de 91 años con una desviación estándar de 20.7). En 239 (47\%) pacientes no se observaron alteraciones. Los principales hallazgos fueron los siguientes: 226 (45\%) casos con pérdida ósea de proceso alveolar, seguido de hallazgos radiográficos sugestivos de alteración en la zona articular (ATM) con 171 (34\%) casos; en 151 (30\%) casos se observaron signos radiográficos de calcificación del ligamento estilohioideo (CLE-síndrome de Eagle) (Figura 1); 18 (3\%) casos presentaron estructuras correspondientes a calcificación de carótida (CC) y en 11 (2\%) casos se localizaron zonas radiolúcidas semejantes a lesiones quísticas (Figura 2). Los hallazgos se reportan en la Tabla 1.

Del resultado de los 226 con PPA, 158 corresponden al género femenino y 68 al masculino, de los cuales, según su expediente clínico, 119 contaban con historial de alguna enfermedad sistémica.

Respecto a la muestra de CLE (Figura 3), el expediente clínico de 51 pacientes indica que presentaron síntomas relacionados con síndrome de Eagle (SE), mismos que se describen en la Figura 4. Otras de las patologías encontradas fueron las calcificaciones de la carótida en 18 casos (Figura 5), sin que se asociaran con alguna manifestación clínica, o hubieran sido detectadas en el expediente Clínico. ${ }^{7,8}$

\section{DISCUSIÓN}

Es la primera vez que se realiza un análisis de radiografías panorámicas en la región de Chihuahua. Además existen pocos estudios con este número de muestra que involucre anexos y estructuras de cavidad oral.

Son impactantes los resultados obtenidos en este estudio, ya que $46 \%$ de la población estudiada contaba

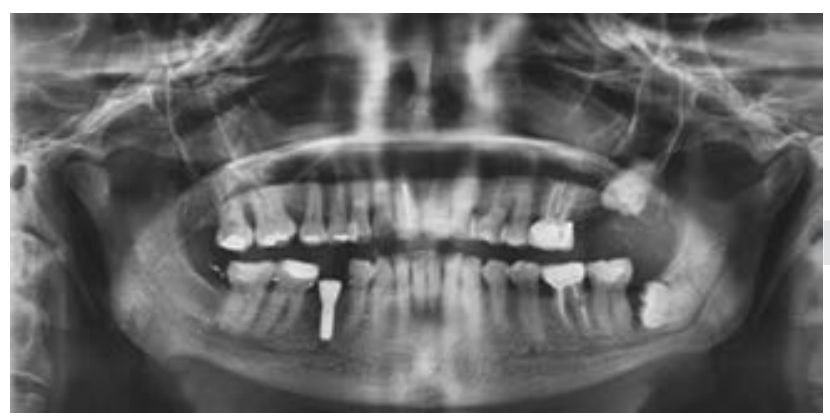

Figura 3: Se visualiza asimetría en ambos cóndilos mandibulares, así como calcificación del ligamento estiloides.
Sintomatología en pacientes con calcificación del ligamento estilohioideo (CLE-síndrome de Eagle)

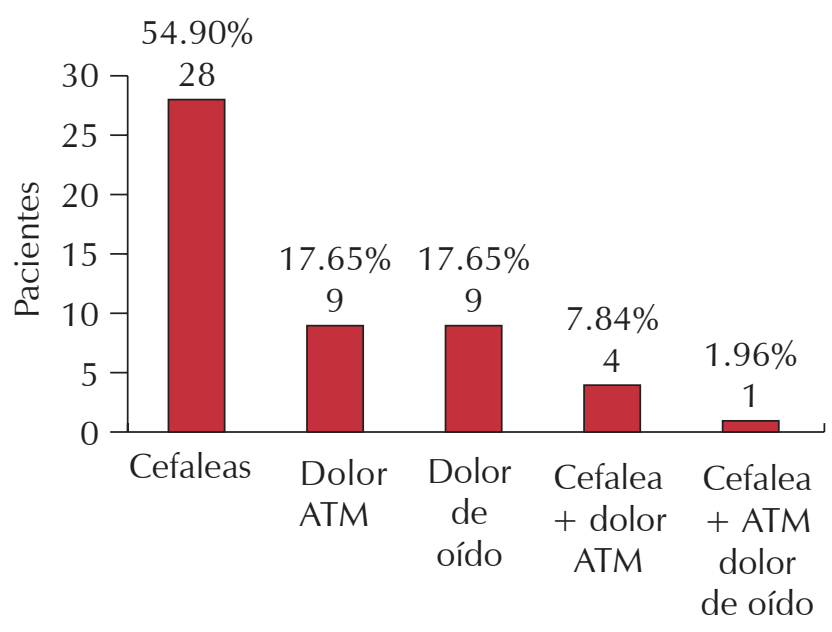

Figura 4: Relación de calcificación del ligamento estilohioideo y expediente clínico.

$\mathrm{ATM}=$ alteración en la zona articular.

con una alteración, del cual un alto porcentaje no se habría reportado por estar asintomático o desconocer las características radiográficas.

Por otro lado, relacionado con la pérdida del proceso alveolar encontramos la periodontitis, la cual se define como «una enfermedad inflamatoria de los tejidos de soporte de los dientes causada por microorganismos o grupos de microorganismos específicos que producen la destrucción progresiva del ligamento periodontal y el hueso alveolar con formación de bolsa, recesión o ambas».${ }^{9}$ Parte de su diagnóstico se basa en la exploración radiológica de los tejidos periodontales y su inspección de tejidos blandos y duros, relacionándose en algunas ocasiones con manifestación de enfermedades sistémicas como cardiovasculares y diabetes mellitus. ${ }^{10,11}$

Uno de los hallazgos más relevantes fue el CLE, se presentó en nuestro estudio en 34\% de los individuos, con predilección por el género femenino, del total con CLE, $34 \%$ de estos pacientes manifestaron en su expediente electrónico haber presentado sintomatología relacionada con síndrome de Eagle.

Existen estudios similares en diferentes poblaciones, pero con un objetivo diferente, por ejemplo: en un estudio que se realizó en una población brasileña concluyeron que en $43 \%$ de la muestra en estudios de radiografías panorámicas digitales se presentó elongación de apófisis estiloides. $^{12}$ 


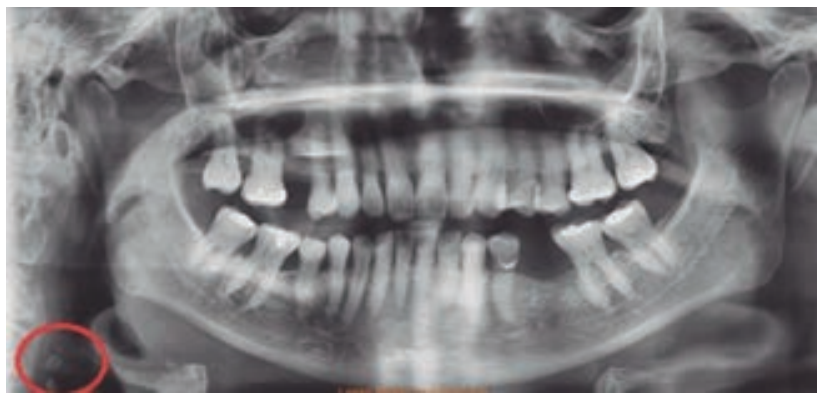

Figura 5: Visualizamos en el lado inferior derecho las calcificaciones a nivel de la carótida. La pérdida del nivel del proceso alveolar es evidente.

A pesar de la alta incidencia de probable SE en nuestro estudio, se requieren análisis y estudios más amplios de manera independiente para su discusión.

Harris \& Clarck $^{13}$ describieron en un estudio de 1,700 radiografías panorámicas una frecuencia de 3.7\% de dientes supernumerarios en contraste con $2 \%$ que encontramos en nuestro estudio. ${ }^{4,5}$

\section{CONCLUSIONES}

Todos estos estudios han presentado mayor relación con las estructuras involucradas directamente con la cavidad oral, y pocos con sus anexos. Al ampliar el campo de observación en esta muestra se ha encontrado un aumento en el porcentaje de hallazgos como los mencionados anteriormente. Es una alerta para los odontólogos y pasantes de odontología que deberán estar entrenados para el análisis de una RP, ya que es una importante herramienta diagnóstica en la práctica profesional no sólo para el propio odontólogo, sino que puede alertar sobre otro tipo de patologías y remitir así al paciente al especialista adecuado para un tratamiento oportuno.

\section{REFERENCIAS}

1. Jiménez OJL, Herrera SJC, Jiménez OJD, Pérez LE, Murillo RJA. Hallazgos incidentales en ortopantomografías o RP maxilomandibulares de pacientes adultos jóvenes. Rev ADM. 2017; 74 (1): 25-31.
2. World Healt Organization. Communicating radiation risks in pediatric imaging: information to support health care discussions about benefit and risk. Organización Mundial de la Salud. 2016; 21.

3. Choi JW. Assessment of panoramic radiography as a national oral examination tool: review of the literature. Imaging Sci Dent. 2011; 41 (1): 1-6.

4. Aguilar O, Gallego GCL, Iriarte AAM, Quintero IS. Prevalencia de hallazgos en radiografías panorámicas de rutina. Rev Nal Odo UCC. 2009; 9 (5): 15-20.

5. Sánchez B, De la Fuente J, Díaz J, Vilar G. Alterations and pathologies prevalence in panoramic radiographs in patients attending the university dental clinic. Int J Odontostomt. 2013; 7 (1): 47-52.

6. Herrera A. La sistematización en la interpretación radiográfica en odontología y su aplicación a estudios intraorales. Colom Med. 2001; 31 (1): 126-129.

7. Kamikawa RS, Pereira MF, Fernandes A, Meurer MI. Estudio de la localización de radiopacidades similares a carótida ateroma calcificada por medio de radiografía panorámica. Oral Surg Oral Med Oral Pathol Oral Radiol Endod. 2006; 101 (3): 374-378.

8. Constantine S, Roach D, Liberali S, Kiermeier A, Starkar P, Janner J et al. Carotid artery calcification on orthopantomograms (CACO Study) - is it indicative of carotid stenosis? Aust Dent J. 2019; 64 (1): $4-10$

9. Zokaris N, Siska I, Natsis K, Piagkou M, Lazaridis N, Skolka A et al. Investigation of the styloid process length in a Greek population. Folia Morphol (Warsz). 2018; 73 (2): 378-388.

10. Caton JG, Armitage G, Berglundh T, Chapple ILC, Jepsen S, Kornman KS et al. A new classification scheme for periodontal and periimplant diseases and conditions - Introduction and key changes from the 1999 classification. J Clin Periodontol. 2018; 89 (1): 1-8

11. López S, Diz-Iglesias P, Seoane-Romero JM, Quintas V, MéndezBrea F, Varela-Centelles P. Actualización en medicina de familia: patología periodontal. SEMERGEN - Medicina de Familia. 2016; 43 (2): 141-148.

12. Hettiarachchi PVKS, Jayasinghe RM, Fonseka MC, Jayasinghe RD, Nanayakkara CD. Evaluation of the styloid process in a Sri Lankan population using digital panoramic radiographs. J Oral Bio and Cran. 2019; (9): 73-76.

13. Harris EF, Clark LL. An epidemiological study of hyperdontia in American blacks and whites. Angle Orthod. 2008; 78 (3): 460-465.

Conflicto de intereses: Los autores declaran no tener conflicto de intereses.

Aspectos éticos: Ninguno.

Financiamiento: No se recibió ningún financiamiento.

Correspondencia:

Nydia Hinojos Villalobos

E-mail: nydia_hinojos@hotmail.com 